\title{
Las posibilidades educativas del paradigma hipertextual: modelos y resultados de investigación
}

\section{The Educational Possibilities of the Hypertext Paradigm: Models and Research Findings}

Concepción López-Andrada ${ }^{1}$

Citation/ Para citar este Artículo: López-Andrada, C. (2020). Las posibilidades educativas del paradigma hipertextual: modelos y resultados de investigación. Colomb. Appl. Linguistic. J., 22(1), pp. 29-39.

Received: 22-Feb.-2019 / Accepted: 26-Mar.-2020

DOI: https://doi.org/10.14483/22487085.14515

\section{Resumen}

Este artículo indaga en modelos y resultados de investigaciones que se han originado a partir de la necesidad creciente por el análisis experimental sobre el hipertexto y su potencia educativa. El conjunto de investigaciones que han sido revisadas en este trabajo ofrece un marco común que, por un lado, se extiende en un contexto institucional, y, por otro lado, contiene lo digital como medio de difusión de conocimiento que implica dinámicas novedosas y subyacentes. El desarrollo de la competencia lectora y del aprendizaje a través de hipertextos se describe como un campo relativamente reciente, en el cual resulta ineludible buscar utilidad y proyección de los resultados para favorecer el aprendizaje de los estudiantes a través de un uso eficiente del hipertexto con fines didácticos, entendido este como una forma compleja de categorizar información y significados.

Palabras clave: competencia lectora, comprensión lectora, estrategias lectoras, hipertexto, tecnologías de la información y comunicación

\begin{abstract}
This paper explores models and research findings that have originated from the growing need for experimental analysis on hypertext and its educational potential. The research that have been reviewed in this work offer a common framework that, on the one hand, extends in an institutional context; and on the other hand, contains what digital is as a means of diffusing knowledge implying novel and underlying dynamics. The development of reading competence and learning through hypertexts is described as a relatively recent field, in which it is inescapable to seek utility and projection of results to promote student learning through efficient use of hypertext for didactics. This concept of didactics is relevant as it is understood as a complex way to categorize information and meanings.
\end{abstract}

Keywords: educational hypertext, information and communication technologies, reading comprehension, reading literacy, reading strategies

1 Centro de Estudios Latinoamericanos de Educación Inclusiva (CELEI, Chile) y Universidad de Extremadura (UEX, España). ORCID (D) https://orcid.org/0000-0002-9423-0434. clopezc@unex.es 


\section{Introducción. Sociedad de información y TIC en educación}

La aceleración es un rasgo que caracteriza el mundo que hoy se habita. La velocidad se manifiesta como una relación de fenómenos del tardocapitalismo y como modo de mediatización de la experiencia a través de la ilusión de la inmediatez (Virilio y Lotringer, 2003). De igual modo, la velocidad e inmediatez serán rasgos de lo que suele llamarse información, al igual que la sobreabundancia y el exceso de la misma (Lash, 2005). En tanto, la sociedad ha ganado en complejidad, la institución educativa todavía es reticente a estos medios, manteniendo una estructura y forma tradicional. Siguiendo a Siemens (2006), en esencia no se ha hecho más que "transferir (no transformar) nuestra identidad física a los espacios y estructuras online" (2006, p. 11). Al igual que sucede con otras tecnologías emergentes, la evolución de los procesos de enseñanza-aprendizaje a través de hipertexto ha sufrido su propia fase de desarrollo en un contexto en el cual se componen y organizan nuevos espacios semióticos como es la sociedad de la información.

En este trabajo se examinan modelos y resultados de investigaciones que se han originado a partir de la necesidad y del interés creciente por el desarrollo de análisis experimentales sobre el paradigma del hipertexto y sus posibilidades educativas. El conjunto de investigaciones que han sido revisadas introduce un marco común que, por un lado, se extiende en un contexto institucional, en el cual se presenta lo académico como un nivel superior en el tratamiento de la información, y, por otro lado, contiene lo digital como medio de difusión de conocimiento que implica dinámicas novedosas y subyacentes. En un examen más profundo, se atenderá a los diseños metodológicos e instrumentos validados para la selección de muestra y recogida de datos por las investigaciones aquí presentadas. Finalmente, el procedimiento empleado tratará de describir y comparar los resultados, límites y aportaciones para futuras investigaciones, estableciendo un espacio para la discusión de propuestas y para las conclusiones provisionales.

\section{El hipertexto: definiciones y características}

El desarrollo de la sociedad del conocimiento requiere de sistemas de almacenamiento y reproducción de datos eficientes y perdurables. De este modo, emerge el hipertexto como una forma de estructurar y jerarquizar la información. Siguiendo a Bruguera y Campàs (2007), se advierte que en un hipertexto la lectura no avanzaría como en un libro, sino que el lector puede saltar de un lugar a otro. Pajares (2004) presenta una definición del hipertexto meticulosa, caracterizándolo como:

\begin{abstract}
una estructura de base informática para organizar información que hace posible la conexión electrónica de unidades textuales (de diferente tamaño, categoría y naturaleza) a través de enlaces (links) dentro de un mismo documento o con documentos externos. Requiere la manipulación activa del lector para poder ser leído/utilizado, además de la actividad cognitiva común a cualquier proceso de lectura.

(Pajares, 2004, p. 34)
\end{abstract}

Una de las características del hipertexto es, pues, la facultad por parte del lector de la posibilidad de elección (Bolter, 1991; Landow, 1989). Otra peculiaridad es que un hipertexto carece tanto de principio como de fin, tal y como se ha señalado anteriormente. No posee una sucesión temporal definida, la retroactividad como rasgo definitorio se materializa en "un proceso dinámico y dialéctico continuo en el que el pensamiento se construye y se enriquece sin parar a partir de las nuevas informaciones que se ha de integrar y que selecciona” (Bruguera y Campàs, 2007, p. 17).

Estos procesos requieren una renovación de los paradigmas, ya que el hipertexto implica transformaciones en el modo de pensar el entorno de lectura y aprendizaje, y necesita de una red de conexiones que lo vuelva "real" y lo convierta en un texto accesible. Es posible considerar, entonces, que se está conformando un nuevo tipo de lector con poder, por lo menos con un poder mayor en el acceso e inmediatez que representarían los textos digitales. Considera Leibrandt que "el libro no puede tratarse de forma aislada ya que los textos 
impresos están integrados en una cultura de medios audiovisuales e interactivos" (2012, p. 6); de este modo, lo que la autora llama "cultura hipertextual" es una cultura que se puede definir como integradora en la medida que interconecta diferentes medios, de ahí la necesidad de desvincular la lectura de su tradicional enlace con el libro.

El carácter flexible del hipertexto requiere de un lector activo y crítico. Llegado a este punto, es fundamental cuestionarse si esto funciona ciertamente así, es decir, existen evidencias de que las TIC inciden en un posible cambio de paradigmas, pero ċsuponen una revolución en el concepto de texto y de lector tan clara como defienden algunos autores? Quizá sí sea posible hablar de una nueva actitud del lector respecto a la lectura como un lugar de experiencias multidireccionales (Landow, 2006). El texto digital modifica el modo de aproximación que el lector tradicionalmente tenía respecto al acto de lectura y al acto comunicativo en general (Baehr y Lang, 2019). El peso significativo de la lectura ya no se apoya tanto en un trabajo de interiorización del individuo como en la "interacción" y la "operatividad", dos rasgos que ahora son constitutivos.

El periodo histórico actual se singulariza por ser un tiempo de mutaciones y convergencias dentro de la cultura textual. Varios autores hablan de la transformación más radical desde los tiempos de Gutenberg (Delany y Landow, 2006); sin embargo, no es posible predecir qué consecuencias exactas acontecerán en el futuro debido a que el momento actual resulta todavía incipiente. Desde la invención de la escritura se han buscado técnicas y medios para "incrementar la rapidez y la comodidad en la localización de la información" (Delany y Landow, 2006, p. 41); de igual modo, es evidente que gran parte delos textos electrónicos o digitales o hipertextos funcionan, hoy por hoy, como un suplemento de los textos manuscritos, mecanografiados o impresos.

\section{Investigaciones y estudios sobre el uso y aplicación de hipertextos en educación. Procedimiento de la revisión}

El procedimiento que se ha aplicado en esta revisión se inicia con la búsqueda de investigaciones publicadas entre 2005 a 2015 recogidas en las siguientes bases de datos: ScienceDirect, ERIC y Scopus, las cuales se han localizado como las más pertinentes para encontrar información bibliográfica de valor en el ámbito de las ciencias sociales y la educación. El tratamiento de estas bases de datos bibliográficas puso en marcha un proceso de búsqueda de palabras clave asociadas con el "hipertexto", en inglés "hypertext", siendo este idioma el predominante en los resultados de las bases de datos. Se desecharon investigaciones que contenían este término, pero cuyos estudios se desarrollaban en ámbitos no relacionados directa o tangencialmente con la didáctica, práctica educativa o contextos educativos.

Se observa que el número de investigaciones encontradas a partir de 2010 sobre la temática aumenta notablemente. Se considera pertinente utilizar este rango de años tan amplio debido a que de esta forma se puede examinar una evolución temporal en los diseños metodológicos y resultados obtenidos en estos estudios empíricos, los cuales se caracterizan en sus inicios por un rasgo más genérico e inconsistente, para acabar con el trascurso de los años en análisis experimentales más sólidos, con mayor fundamento teórico y metodológico. Se seleccionaron para esta revisión únicamente artículos publicados en revistas indexadas y de impacto, procedentes del área de las ciencias de la educación, comunicación y humanidades.

Consecuentemente, se evidencian huecos en las investigaciones revisadas, pero también potencialidades cuando se considera el hipertexto como una construcción textual y gráfica que no solo ayuda a la navegación, a la recuperación de información, sino que concierne a los nuevos modos de leer e interaccionar en entornos digitales.

\section{Revisión de evidencias sobre el uso didáctico del hipertexto}

El hipertexto tiene grandes posibilidades en entornos educativos y cabida en muy diversas áreas, a pesar de que su integración y desarrollo 
como herramienta didáctica ha sido escasa ${ }^{2}$. Investigaciones recientes de Walhout et al. (2015) trabajan con entornos de aprendizaje con hipertextos (HLE, por sus siglas en inglés de Hypertext Learning Environments), analizando los efectos y las consecuencias de su desarrollo en relación con el aprendizaje y las competencias. Estos entornos hipertextuales no son lineales, los estudiantes se ven obligados a desplegar formas de navegar a través de ellos. Por lo tanto, el desarrollo de interfaces que faciliten y guíen en la navegación es un aspecto esencial para el aprendizaje, según los autores.

Este estudio evidencia que el éxito de aprendizaje en HLE depende tanto de la predisposición y peculiaridades del alumnado como de las características de estos entornos de aprendizaje con hipertextos. Enlazando con las investigaciones de Shabani, Naderikharaji y Reza (2011), Jeong (2012) y Daniel y Woody (2013), se plantea que a raíz de la paulatina demanda y consecuentemente necesidad de articular y ampliar los límites concernientes a la competencia lectora digital y a la creación y aplicación de hipertextos educativos, será indispensable incidir en las transversalidades de la práctica lectora a través de hipertextos, ya se desempeñe en un ámbito educativo o doméstico. En el estudio realizado por Shabani et al. (2011) se puede distinguir, entre la población de estudiantes de posgrado analizada, aspectos de la conducta lectora tales como: (a) el estilo de los recursos digitales de lectura, (b) la cantidad de anotaciones realizadas, (c) la cantidad de impresiones de documentos digitales e hipertextuales para su lectura, (d) la preferencia de lectura.

Los hipertextos representan una oportunidad para el desarrollo de nuevas habilidades por parte de los lectores, así como nuevas incertidumbres, ya que, en el proceso lector, resulta corriente perder los anclajes de lectura y la propia extensión del texto. El estudio realizado por Evans y Po (2007)

2. Pajares (2004) ilustra sobre la dificultad de encadenar teoría y práctica hipertextuales. Esta idea se concreta en la confluencia entre hipertexto y educación, campo poco estudiado y con escasas, pero destacables, experiencias. Precisamente, la mayoría de propuestas en el campo del hipertexto y la educación proceden de la informática de sistemas. Otro ámbito que se ha interesado por el tema del hipertexto y la educación es el de la psicología cognitiva y la pedagogía, al igual que el área particular del hipertexto y la educación literaria. toca el tema de la fatiga que puede generar en los estudiantes este tipo de textos, pues tienden a cansarse repetidamente con la lectura de textos digitales, inclusive, aspectos como la disposición corporal son estimados por los mismos.

Liu (2005) describe en sus investigaciones los efectos del hipertexto en los procesos de comprensión. El carácter fragmentario de esta forma textual es el rasgo principal que afectaría a los procesos de comprensión lectora. Cabe preguntarse a través de estos estudios si existen evidencias de los efectos del hipertexto en el aprendizaje y comprensión y, seguidamente, si estos efectos son de carácter positivo o negativo. En un primer término y siguiendo con el análisis de Liu (2005) se evidencia que, además de la incertidumbre, los alumnos se sienten abrumados por el carácter inabarcable de la información y por factores relacionados con el manejo de los dispositivos tecnológicos debido a la insuficiente competencia en la resolución de problemas durante la navegación y comprensión de la información. De este modo, otro condicionante importante es la multiplicación de vínculos y enlaces en un documento o navegación.

El lector en la pantalla puede llegar, entonces, a una sensación de aturdimiento ante la información inagotable, tal y como se señaló antes, por lo que tiende a practicar una suerte de "escaneo", es decir, una lectura transitoria y ágil; en el momento en el que algo capta toda su atención, este se sumerge en una lectura profunda. La pantalla no es reconocida como un espacio para el desarrollo de una lectura dilatada y profunda, sino como un lugar donde es posible extraer la información que a posteriori será objeto de lectura en formato impreso (Liu, 2005). Las evidencias sobre cómo la proliferación de textos digitales afecta a la cantidad y la calidad del comportamiento lector se sintetizan en que la concentración y la profundidad de lectura en entornos digitales ha disminuido, con independencia de que los lectores o usuarios pasan actualmente un tiempo considerable leyendo. La lectura en pantalla implicaría un sustancial aumento de la exploración, una tendencia a la búsqueda de las palabras claves de un texto, un seguimiento de los vínculos, sobreviniendo también una lectura menos enfocada a la anotación que la tradicional. 
Las actuales investigaciones sobre lectura enfocan sus objetivos en la consideración y análisis en torno a las incidencias en las prácticas lectoras de las nuevas tecnologías, las cuales han permitido que el proceso lector suceda en cualquier momento y en cualquier sitio mediante el uso de dispositivos (ordenadores, tabletas, teléfonos móviles) que fomentan una lectura no lineal y que excluyen la presencia del documento impreso. Es el caso de Andretta (2005), Abdul y Hasan (2007), y de Maynard y Cheyne (2005). Así, Andretta (2005) examina los desafíos generados por la proliferación de la información digital y la consiguiente necesidad para el sistema educativo de una correcta planificación en alfabetización digital de los alumnos como contrapeso crítico, construyendo una comparativa entre los modelos de Australia, Estados Unidos y Reino Unido.

Por su parte, Abdul y Hasan (2007) intentan arrojar luz sobre los hábitos de lectura y las actitudes de los estudiantes de una licenciatura, teniendo en cuenta la variable de género. El estudio reveló que internet es considerado por los alumnos como una fuente de lectura cada vez más significativa, existiendo una brecha entre los programas académicos y los tipos de materiales y recursos de lectura que se disponen en la red. También se observaron algunas diferencias en los hábitos de lectura y actitudes hacia la misma entre los hombres y mujeres participantes, conclusión que replican otras investigaciones similares. Maynard y Cheyne (2005) consideran la importancia de la investigación de los efectos de la lectura digital a largo plazo en el aprendizaje de los estudiantes y en el particular interés y planificación por parte de docentes, bibliotecarios y padres.

El trabajo de Vörös, Rouet y Pléh (2011) tiene de novedoso la utilización del hipertexto para potenciar tanto las capacidades de memoria como las capacidades visoespaciales. El aprendizaje a través de hipertextos se amplificará con tareas o subtareas de compresión textual y mediante la orientación espacial.

Ostovar-Namaghi y Noghabi (2014) realizan una comparación entre el desarrollo de estrategias metacognitivas de lectura de hipertextos y materiales académicos impresos en un posgrado de ciencias. Los investigadores incluyen tres tipos de estrategias por desarrollar: (a) estrategias de lectura a nivel global, (b) estrategias para la resolución de problemas concretos, (c) estrategias de apoyo de la lectura. Resulta importante destacar que el uso percibido por los estudiantes de las estrategias globales, estrategias para la resolución de problemas y estrategias de apoyo a la lectura de textos impresos, se encontraba en un nivel "moderado", muy similar al correspondiente al texto en línea, a excepción de la particularidad del uso de las estrategias globales, circunscritas a los estudiantes-usuarios con un nivel alto en el desarrollo de estrategias.

Por otra parte, los investigadores Sahin y Alsancak (2011), estudian el efecto producido por hipertextos de muy variada extensión en el desarrollo de estrategias de compresión de alumnos que estudian un idioma. El experimento que se llevó a cabo consistía en la lectura de cuatro textos, dos narrativos y dos informativos, y derivó en que los resultados de las pruebas de comprensión de los estudiantes en el grupo con los hipertextos más extensos fueron más elevados que los del grupo con los textos más cortos y fragmentados.

Zammit (2011) describe a través de la lingüística sistémico-funcional ${ }^{3}$ la construcción de un texto por parte de un grupo de estudiantes durante una sesión de búsqueda sobre un tema determinado. Toma como base el reciente aumento de la utilización de hipertextos para localizar información, por lo que los estudiantes necesitan desarrollar estrategias

\footnotetext{
3. Esta teoría, desarrollada principalmente por Michael Halliday, considera el lenguaje como un sistema de alternativas disponibles. Siguiendo a Ghio y Fernández: "si la teoría pretende postular al aspecto social como parte inherente al sistema lingüístico, no puede limitarse a enumerar sus usos; debe mostrar cuál es la relación dialéctica que se establece entre esos usos sociales y el sistema" (2005, p. 15). De este modo, según los autores, los objetivos de una gramática funcional pueden ser múltiples en relación con sus posibles contextos de aplicación, desde una gramática para uso escolar que no emplea la misma terminología que un lingüista profesional, pasando por la aplicación en lingüística computacional donde la gramática debe dilucidar la relación entre sistema y estructura, hasta el análisis crítico del discurso donde es necesario incorporar una gran cantidad de información contextual para conseguir la deconstrucción ideológica de textos.
} 
de decisión de navegación que contribuyan a la comprensión de los contenidos y temas para evitar que estos mismos se conviertan en incomprensibles o dispersos.

Dos trabajos se presentan fundamentales sobre el estudio de la relación del hipertexto con la comprensión lectora y los procesos de aprendizaje, son las investigaciones realizadas por Salmerón et al. (2005) y Salmerón et al. (2009), cuyos objetivos son el análisis de la relación entre el uso de la estrategia y la comprensión a la pormenorización del empleo de las descripciones gráficas en hipertextos educativos. De esta manera, en Salmerón et al. (2005) se señala la contradicción entre los resultados arrojados por las investigaciones sobre la temática, explorando el probable factor que afectaría a este hecho, causado por el no control de los efectos en la comprensión de las estrategias de lectura en hipertextos. Como consecuencia, los autores afirman que las estrategias de lectura en hipertextos son la regla de decisión que un lector sigue cuando navega a través de los diferentes nodos de un hipertexto, así, por ejemplo, los lectores pueden leer a través de los contenidos y selección de nodos con contenidos de interés o relación con los párrafos anteriormente leídos.

Las estrategias de lectura afectarán la cantidad de información adquirida y al orden de la lectura. Estas dos características textuales tienen un impacto en la representación del texto incorporado por el lector. Concretamente, los autores proponen que la cantidad de información leída por un lector afecta a la llamada base textual, además de la significación del orden seguido por dicho lector.

En otro estudio, Salmerón et al. (2009) delimitan sus investigaciones al análisis de la compresión a través de las descripciones gráficas en hipertextos educativos, caracterizadas por transmitir la estructura del texto esquemáticamente con el objeto de fomentar la comprensión de los contenidos. De esta manera, los autores exploran la manera en la que las descripciones gráficas en hipertextos afectan a la comprensión respecto al momento en que los estudiantes leen para conseguir una visión de conjunto y los inconvenientes implicados con la lectura de hipertextos. Los resultados de dos estudios experimentales de movimiento ocular durante la lectura revelaron que los alumnos que utilizan estrategias de lectura profunda y que son lectores competentes en papel, serán buenos lectores de hipertextos, siempre que ahonden en la compresión de los contenidos y vayan más allá de la visión global detectada en la lectura de las ideas principales y del scanning, es decir, la exploración de una parte del contenido sin la necesidad de leer todo.

Un estudio muy llamativo es el que realiza Bromme y Stahl (2005), pues examinan el impacto y arraigo de las distintas metáforas alrededor de la idea y el proceso de construcción de un hipertexto. De este modo, dos grupos de veinte estudiantes universitarios sin experiencia en el diseño de esta herramienta recibieron explicaciones introductorias referidas a la forma de la metáfora del libro y a la forma de la metáfora del espacio, analizando el impacto en los procesos de construcción de hipertexto, y en el aprendizaje en los estudiantes universitarios que producen hipertextos. A la mitad de los estudiantes se les aclaró la idea de hipertexto como una metáfora del libro y a la otra mitad como una metáfora de espacio, tanto un grupo como otro, enlazaron y construyeron nodos previamente preparados sobre el tema "internet" y fueron grabados en vídeo, solicitándoles durante su trabajo que informaran sobre lo que estaba aconteciendo.

Los investigadores observaron que la metáfora del libro estimula una visión mucho más lineal de percibir los hipertextos, entrando en conflicto con la complejidad de los contenidos por procesar; la metáfora del espacio permite una correspondencia entre las estructuras semánticas complejas y las estructuras de hipertexto complejas. Por lo tanto, la metáfora espacial parece ser la más apropiada para explicar el hipertexto a los estudiantes, ya que resultó más útil a la hora de que los estudiantes se enfrentaran a la complejidad de las estructuras de contenidos y a las estructuras hipertextuales. No obstante, el rendimiento en la prueba de conocimientos de los alumnos reveló que el uso de la idea de "metáfora" a la hora de construir un hipertexto no garantiza que los procesos de aprendizaje sean más profundos.

Finalmente, Chen y Yen (2013) amplían los estudios sobre la influencia de las anotaciones con 
hipertextos en la competencia y comprensión lectora de los alumnos en el aprendizaje de vocabulario de idiomas extranjeros y de las actitudes de los usuarios hacia la presentación de anotaciones. Se recogieron datos de ochenta y tres estudiantes universitarios cuya lengua materna no era el inglés. Los participantes leían pasajes con distintos formatos de anotación como se mencionan a continuación.

- El texto de anotación: consideradas notas explicativas adicionales e incrustadas dentro de un texto junto a la palabra anotada. No era necesario la acción del ratón para recuperar el significado de las palabras.

- Glosario de anotaciones: lista de definiciones para cada palabra anotada que aparece al final o al margen del texto. Es necesaria la acción del clic con el ratón sobre la palabra vinculada para llegar al glosario.

- Anotaciones emergentes: notas explicativas adyacentes a cada palabra anotada. Las anotaciones emergentes son visibles solo cuando se hace clic con el ratón sobre la misma.

Los resultados indican que se alcanza el peor rendimiento en la comprensión lectora con el formato "texto de anotación". Así, se observó que el mejor rendimiento fue en la condición de "anotaciones emergentes". Los autores destacan, entonces, que para la adquisición de vocabulario el uso de hipertextos beneficia significativamente el aprendizaje entre alumnos con medias y altas competencias. Los hallazgos de la investigación proporcionan interesantes directrices para la planificación y diseño de la lectura digital.

\section{Discusión y proyección para futuras investigaciones}

Las investigaciones revisadas en el apartado anterior poseen un carácter empírico de gran validez, cuyos resultados se pueden complementar dependiendo de diversos grados. La recogida de datos se llevó a cabo a través de la observación, la creación de grupos controlados de alumnos o a través de cuestionarios y encuestas. También se evidencia un estado preliminar en muchas de las investigaciones elaboradas, aportando, en algunas ocasiones, conclusiones discordantes. Esto es indicado y justificado por los propios investigadores. Precisamente, las reducidas muestras manejadas, la fundamentación a partir de un único instrumento introducido de forma aislada y la obtención de datos apoyados únicamente en las opiniones y preferencias de los participantes, se añaden al breve recorrido y escasa incidencia de este tipo de estudios. Los límites para la investigación son señalados en la mayoría de los trabajos; estas barreras se convierten en pistas que deberán ser soslayadas en futuras investigaciones.

Es posible hablar de tres núcleos esenciales a la hora de aproximarse a la práctica lectora con hipertextos:

- Los conocimientos previos de los lectores.

- La capacidad de comprobación o autorregulación, algo que es posible llamar "conciencia lectora".

- El propósito y objetivos de la lectura. Respondería a las preguntas: ¿para qué se lee?, ¿qué tipo de información y contenidos busca el lector?

Desde la perspectiva de una nueva cultura textual necesaria para los lectores que tienen que hacer frente a los diferentes problemas y dificultades que surgen del entorno de la lectura hipertextual (Vörös et al., 2011; Walhout et al., 2015; Evans y Po, 2007; Ostovar-Namaghi y Noghabi, 2014), será necesario incorporar nuevas habilidades y competencias que es posible asociar a cinco campos: (a) la identificación de problemas y preguntas, (b) la localización de información de múltiples recursos en línea, (c) la evaluación crítica de la información en línea, (d) la síntesis de la información en línea, (e) la comunicación y el intercambio de información en línea (Salmerón et al., 2005).

La investigación ha llevado a destacar el papel de las estrategias (Ostovar-Namaghi y Noghabi, 2014; Salmerón et al., 2005; Zammit, 2011) en la práctica de tareas de lectura en la red. Las estrategias 
de lectura se caracterizan por su sistematicidad y buscan (ya sea la lectura en pantalla o en papel) decodificar un mensaje y construir significados. A partir de los estudios clásicos sobre desarrollo de estrategias en texto impreso (Tierney y Readence, 1985) se ha llegado a conocer que los lectores expertos aplican las estrategias de forma flexible y proyectan los ajustes que consideran adecuados para conseguir sus objetivos.

La investigación del proceso lector en entornos hipertextuales enmarca la comprensión de lectura en internet como una sucesión que implica el uso de estrategias para generar preguntas importantes $\mathrm{y}$, posteriormente, localizar, evaluar críticamente, sintetizar y comunicar información que ayude a responder dichas preguntas (Ziming, 2005). Emergen diversas estrategias que el lector usa para leer en pantalla; de esta manera, se evidencia que los lectores competentes diferencian entre la información específica y la adquisición de conocimiento general. La cantidad de estrategias desarrolladas, asimismo, es distinta según el propósito (Zammit, 2011). Así, los lectores utilizarán un menor número de estrategias cuando el propósito es el entretenimiento que cuando están buscando adquirir conocimientos generales o información específica. También se evidencia que los lectores entrecruzan nuevas estrategias inherentes al hipertexto junto a las que adaptan provenientes de las utilizadas en un texto impreso.

Tal y como se ha reiterado en los apartados anteriores, las investigaciones vinculadas a la lectura y aprendizaje a través de hipertextos pertenecen a un escenario relativamente reciente. Por consiguiente, es reseñable la indagación en aspectos como la utilidad y proyección de los resultados para favorecer el aprendizaje de los estudiantes en cualquier nivel educativo, particularmente, analizar de qué forma interactúan estos elementos para explicar mejor el desempeño en tareas de lectura y de desarrollo de competencia lectora, lo que consecuentemente aportaría conocimientos valiosos que se podrían aplicar a distintos contextos y se traducirían en el uso eficiente del hipertexto con fines didácticos, entendido este como una forma práctica y eficaz de estructurar y categorizar información y significados complejos.

\section{Conclusiones provisionales}

La exigencia y celeridad de estos tiempos necesitan de un perfil crítico que medie desde la institución educativa, pero también que tenga en cuenta que hoy por hoy el conocimiento y los procesos de formación se producen en muy distintos y variados contextos.

La ruptura de la linealidad característica de los textos digitales e hipertextos puede ser un recurso muy estimulante a la hora de trabajar con diversos discursos y géneros textuales en el aula. Los estudiantes tienen la posibilidad de revisar diversos contextos, voces y fragmentos en los que cabe la posibilidad de habitar nuevos mundos digitales alejados de los espacios comunes y normativos, donde las fronteras de la textualidad tradicional tienden a borrarse. El hipertexto como texto sin inicio ni fin, como texto más abismal y fragmentado que lineal, busca a un lector flexible, versátil y activo.

A la luz de la discusión anterior, se puede señalar que existen dos tipos de conocimiento previo que al parecer tienen un papel relevante en la comprensión lectora en internet: el conocimiento previo sobre el tema y el conocimiento previo sobre el uso del internet. El papel de ambos ha sido valorado al resolver una tarea específica en el texto de internet. La relación entre los distintos tipos de conocimiento previos y entre los distintos tipos de estrategias (lectoras y digitales) es esencial, a pesar de que todavía no existe suficiente evidencia concluyente que explique los vínculos que poseen estos conocimientos previos con el pensamiento estratégico al leer en internet (Morales, Flores y Meza, 2017). De esta forma, y como se ha visto en los apartados anteriores, se produce en algunas investigaciones el resultado provisional de mejora por parte de lectores con dificultades en el texto impreso que alcanzan mejores resultados con la lectura digital.

En futuros trabajos, será necesario responder a la cuestión de hasta qué punto este conocimiento previo tiene que ver con las habilidades digitales comunes que se articulan en las prácticas lectoras en internet, de igual modo que puede darse por parte del lector un buen desarrollo de ciertas 
estrategias de comprensión lectora para la búsqueda y localización de información en la red, pero no de otras estrategias relacionadas con la evaluación y comunicación de información (Keck, Kammerer y Starauschek, 2015; Morales et al., 2017).

Las recientes revisiones exploran los elementos tanto potenciadores como problemáticos de la lectura digital y de la lectura en papel (Singer y Alexander, 2017). También se aborda la dicotomía entre texto lineal y no lineal. De este modo, los buenos lectores de texto lineal seleccionarán y trabajarán con páginas de hipertexto y webs más relevantes que los etiquetados como lectores débiles o lectores con menos competencia lectora (Hahnel et al., 2016).

Otros estudios recientes se replantean la figura del nativo digital en su relación con los procesos de enseñanza y aprendizaje a través de hipertextos (Fajardo, Villalta y Salmerón, 2016), dando a conocer una síntesis muy ajustada de la competencia lectora digital cuyo foco hay que situarlo tanto en el desarrollo de la comprensión lectora, como en el uso, la reflexión y el disfrute de los textos escritos con el propósito de conseguir el objetivo planteado, pero también el buscar un autoconocimiento y la participación activa en la sociedad. Concretamente, este estudio establece un vínculo muy estrecho entre el desarrollo de la competencia digital y la localización, la integración y la reflexión sobre la información que aparecen en los hipertextos. Los autores asumen que desde los currículos oficiales será necesario activar programas educativos que tengan en cuenta el desarrollo de las habilidades digitales necesarias como la búsqueda de información en redes o el uso de hipervínculos.

Otros trabajos que revisan diversas investigaciones y analizan la lectura digital han aparecido en los últimos años, como es el caso del completo enfoque articulado por Walsh (2016), donde se establecen varias categorías que a nivel orientativo resultan especialmente válidas para encaminar los aportes al campo de estudio: la teoría de la lectura; comprensión lectora; el efecto de las herramientas, plataformas y diseños de un documento; lectura en pantalla versus lectura en papel. Esta revisión se centra especialmente en el papel de las bibliotecas universitarias y en propuestas para la comunidad académica, de tal modo que dependerá de estos espacios académicos y de su planificación el facilitar el acceso a medios, bases de datos y documentos impresos y electrónicos, el proporcionar espacios para una reflexión profunda y una administración actualizada de fondos $y$ colecciones.

Tratándose de un campo relativamente nuevo, en investigaciones vinculadas a la lectura en internet se manifiestan limitaciones en relación con el número de investigaciones analizadas que se ajustan a una búsqueda temporal determinada de cinco años. De igual modo, será fundamental atender a aspectos socioeducativos y pragmáticos de estas prácticas en entornos virtuales, es decir, al desarrollo de redes y comunidades, junto a los cruces de formatos y de textualidades dependiendo de cada situación comunicativa.

Por otro lado, será imperativo buscar utilidad de los resultados para favorecer el aprendizaje de los estudiantes en cualquier nivel educativo. Particularmente, entender en qué forma interactúan estas variables para explicar mejor el desempeño en tareas de lectura aportaría un conocimiento valioso que eventualmente se traduciría en el uso eficiente de un medio tan poderoso como lo es internet.

\section{Referencias}

Abdul, N. y Hasan, A. (2007). Reading habits and attitude in the digital age. The Electronic Library, 25(3), 285298. http://dx.doi.org/10.1108/02640470710754805

Andretta, S. (2005). From prescribed reading to the excitement or the burden of choice. Aslib Proceedings, 57(2), 181-190. https://doi. org/10.1108/00012530510589146

Baehr, C. y Lang, S. (2019). Hypertext theory: theoretical foundations for technical communication in the 21st century. Technical Communication, 66(1), 93-104.

Bolter, J. (1991). Writing space: The computer, hypertext, and the history of writing. Lawrence Erlbaum Associates.

Bromme, R. y Stahl, E. (2005). Is a hypertext a book or a space? The impact of different introductory metaphors on hypertext construction. Computers 
and Education, 44(2), 115-133. https://doi. org/10.1016/j.compedu.2004.01.003

Bruguera, E. y Campàs, J. (2007). El hipertexto y los blogs. Editorial UOC.

Chen, I. y Yen, J. (2013). Hypertext annotation: Effects of presentation formats and learner proficiency on reading comprehension and vocabulary learning in foreign languages. Computers and Education, 63, 416-423. https://doi.org/10.1016/j. compedu.2013.01.005

Daniel, D. y Woody, W. (2013). E-textbooks at what cost? Performance and use of electronic v. print texts. Computers and Education, 62, 18-23. https://doi. org/10.1016/j.compedu.2012.10.016

Delany, P. y Landow, G. (2006) El texto en la época de la reproducción electrónica, en Teoría del hipertexto: La literatura en la era electrónica. Arco Libros.

Evans, E., y Po, J. (2007). A break in the transaction: Examining students' responses to digital texts. Computers and Composition, 24(1), 56-73. https:// doi.org/10.1016/j.compcom.2006.12.003

Fajardo, I., Villalta, E. y Salmerón, L. (2016). ¿Son realmente tan buenos los nativos digitales?: relación entre las habilidades digitales y la lectura digital. Anales de psicología, 32(1), 89-97. http:// dx.doi.org/10.6018/analesps.32.1.185571

Ghio, E. y Fernández, M. (2005). Manual de lingüística sistémico funcional: el enfoque de MAK Halliday y R. Hassan: aplicaciones a la lengua española. Universidad Nacional del Litoral.

Hahnel, C., Goldhammer, F., Naumann, J. y Kröhne, U. (2016). Effects of linear reading, basic computer skills, evaluating online information, and navigation on reading digital text. Computers in Human Behavior, 55, 486-500. https://doi.org/10.1016/j. chb.2015.09.042

Jeong, H. (2012). A comparison of the influence of electronic books and paper books on reading comprehension, eye fatigue, and perception. The Electronic Library, 30(3), 390-408. http://dx.doi. org/10.1108/02640471211241663

Keck, D., Kammerer, Y. y Starauschek, E. (2015). Reading science texts online: Does source information influence the identification of contradictions within texts? Computers and Education, 82, 442-449. http://dx.doi.org/10.4304/jltr.5.4.865-87210.1016/j. compedu.2014.12.005

Landow, G. (1989). Hypertext in literary education, criticism, and scholarship. Computers and the Humanities, 23(3), 173-198.
Landow, G. (2006). Hypertext 3.0: Critical theory and new media in an era of globalization. Johns Hopkins University Press.

Lash, S. (2005). Crítica de la información. Amorrortu.

Leibrandt, I. (2012). De la competencia alfabética a la competencia hipertextual. En A. Mendoza y J. Amo (Coords.). Perspectivas en investigación $e$ innovación didáctica en recepción lectora. Leer hipertextos. Editorial Universidad de Almería.

Maynard, S. y Cheyne, E. (2005). Can electronic textbooks help children to learn? The Electronic Library, 23(1), 103-115. https://dspace.lboro.ac.uk/2134/3624

Morales, M., Flores, R. y Meza, J. (2017). El papel de las estrategias y los conocimientos previos al leer en Internet: revisión y discusión de la literatura relevante al tema. Avances en Psicología Latinoamericana, 35(1), 141. http://dx.doi. org/10.12804/revistas.urosario.edu.co/apl/a.3624

Ostovar-Namaghi, S. y Noghabi, A. (2014). A comparison of perceived use of the metacognitive reading strategies by Iranian Master of Science students for hypertext and printed academic materials. Journal of Language Teaching and Research, 5(4), 865-872. https://doi.org/10.4304/jltr.5.4.865-872

Pajares, S. (2004). Literatura digital. El paradigma hipertextual. Universidad de Extremadura.

Sahin, A. y Alsancak, D. (2011). The effect of hypertexts with different lengths on reading and comprehension skills of the students. Procedia. Social and Behavioral Sciences, 28, 347-354. http://dx.doi.org/10.1016/j. sbspro.2011.11.066

Salmerón, L., Baccino, T., Cañas, J., Madrid, R. y Fajardo, I. (2009). Do graphical overviews facilitate or hinder comprehension in hypertext? Learning with ICT: New perspectives on help seeking and information searching, 53(4), 1308-1319. http:// dx.doi.org/10.18239/ocnos 2017.16.2.1287

Salmerón, L., Cañas, J., Kintsch, W. y Fajardo, I. (2005). Reading strategies and hypertext comprehension. Discourse Processes, 40(3), 171-191.

Shabani, A., Naderikharaji F. y Reza, M. (2011). Reading behavior in digital environments among higher education students. Library Review, 60(8), 645-657. http://dx.doi.org/10.1108/00242531111166683

Siemens, G. (2006). Knowing knowledge. Lulu. com.

Singer, L. y Alexander, P. (2017). Reading across mediums: Effects of reading digital and print texts on comprehension and calibration. The journal of experimental education, 85(1), 155-172. http:// dx.doi.org/10.1080/00220973.2016.1143794 
Tierney, R. y Readence, J. (1985). Reading strategies and practices. A compendium. Pearson.

Virilio, P. y Lotringer, S. (2003). Amanecer crepuscular. Fondo de Cultura Económica.

Vörös, Z., Rouet, J. y Pléh, C. (2011). Effect of high-level content organizers on hypertext learning. Computers in Human Behavior, 27(5), 2047-2055. https://doi. org/10.1016/j.chb.2011.04.005

Walhout, J., Brand-Gruwel, S., Jarodzka, H., Van Dijk, M., De Groot, R. y Kirschner, P. (2015). Learning and navigating in hypertext: Navigational support by hierarchical menu or tag cloud? Computers in Human Behavior, 46, 218-227. https://doi. org/10.1016/j.chb.2015.01.025
Walsh, G. (2016). Screen and paper reading research. A literature review. Australian Academic and Research Libraries, 47(3), 160-173. https://doi.org/10.1080/00 $\underline{048623.2016 .1227661}$

Zammit, K. (2011). Moves in hypertext: The resource of negotiation as a means to describe the way students navigate a pathway through hypertext. Linguistics and Education, 22(2), 168-181. https://doi. org/10.1016/j.linged.2011.02.005

Ziming, L. (2005). Reading behavior in the digital environment: Changes in Reading Behavior Over the Past Ten Years. Journal of Documentation, 61(6), 700712. https://doi.org/10.1108/00220410510632040 